Z. Klin. Chem. Klin. Biochem.

11. Jg. 1973 , S. $215-219$

\title{
Untersuchungen zur Thrombocytenadhäsivität bei Patienten mit Gefäßkrankheiten
}

\author{
Von H. Köstering, Ch. Walther, M. Grüne, F. König und P. Völker \\ Aus der Medizinischen Univ.-Klinik, Göttingen
}

(Eingegangen am 4. Oktober 1972/28. Februar 1973)

\begin{abstract}
Zur Bestimmung der Thrombocytenadhäsivität bei verschiedenen Patientengruppen mit Hypertonie, Adipositas, Diabetes mellitus, Myocardinfarkt, Venenthrombosen und akuten thrombembolischen Gefäßkrankheiten wurde der SALZMAN-Test angewandt.

Die Untersuchungsergebnisse bei 8 Patienten mit verschiedenen Krankheiten ohne Hinweise auf Gefäßbeteiligung entsprachen, ähnlich wie bei 17 Patienten mit einer kurzfristig bekannten Hypertonie und 9 Patienten mit älteren Thrombosen, den bei 58 Normalpersonen gefundenen Mittel werten. Demgegenüber war die Differenz der gemessenen Thrombocytenadhäsivität zwischen dem Mittel wert der Gruppe der Normalpersonen und den Mittelwerten von 30 Personen mit länger bestehender Hypertonie, 19 Patienten mit Adipositas, 41 Personen mit Diabetes mellitus, 34 Patienten mit Myocardinfarkt, 18 Patienten mit Angina pectoris sowie 12 Paticnten mit akuten Venenthrombosen und akuten thrombembolischen Gefäßkrankheiten statistisch signifikant. Aus den erhobenen Befunden folgt, daß die mit dem SAL\%MAN-Test erfaßte Thrombocytenadhäsivität bei den zuletzt genannten Patientengruppen signifikant $(p=<0,001)$ gesteigert ist.
\end{abstract}

\section{Studies on the adhesive capacity of thrombocytes in patients with afflictions of the circulatory system}

SalzMan's test was used to determine the adhesive capacity of the thrombocytes in various groups of patients suffering from hypertension, obesity, diabetes mellitus, myocardial infarction, venous thrombosis and acute thromboembolic diseases of the blood vessels.

The results obtained in 8 patients who suffered from various diseases but showed no indication of an affliction of the blood vessels corresponded with the mean values found in 58 normal persons. Investigations on 17 patients with recently diagnosed hypertension and 9 patients with long standing thrombosis showed similar results. In contrast, the difference between the mean values of measured adhesive capacity of the thrombocytes in the group of normal persons and the mean values found in 30 patients with long standing hypertension, 19 patients with obesity, 41 patients with diabetes mellitus, 34 patients with myocardial infarction, 18 patients with angina pectoris and 12 patients with acute venous thrombosis and acute thromboembolic diseases of the blood vessels was statistically significant $(p=<0.001)$. It follows from these results that the adhesive capacity of the thrombocytes as measured with SALzMAN's test is significantly increased in the last mentioned groups of patients.

Die Bedeutung von corpusculären Bestandteilen des Blutes, der Thrombocyten, für die Blutgerinnung und für die Entstehung von Thromben ist seit langem bekannt (1-3). Umstritten ist jedoch noch immer, ob die Thrombocyten und deren Funktionsänderungen in der Pathogenese der Atherosklerose eine entscheidende Rolle spielen (4-6). Hierbei liegt die Hauptschwierigkeit im Fehlen von verläßlichen und allgemein akzeptierten Untersuchungsmethoden zur Messung der verschiedenen Thrombocytenfunktionen. $Z$ war sind inzwischen viele Tests entwickelt worden $(7-20)$, die teilweise sehr aufwendig sind $(16-20)$ oder aber nur in der Hand des damit sehr Erfahrenen verläßliche und eindeutige Ergebnisse liefern. Erst kürzlich wurde wiederum die ganze Problematik verschiedener Thrombocytenfunktionsuntersuchungen deutlich (21), als es im Rahmen eines Ringversuches nur wenigen Untersuchern gelang, eine erniedrigte Thrombocytenfunktion sicher $\mathrm{zu}$ erfassen. Wir wählten nach Versuchen mit einigen anderen Untersuchungsmethoden für unsere Untersuchungen das Testmodell von SalzmaN $(22,23)$, das dem Hellem II-Test ähnlich ist, da beide keine Anticoagulantien oder decalcifizierende Substanzen vor der Adhä̈sivitätsmessung benötigen und andererseits der Kontakt des Blutes mit der Glasoberfläche ohne Manipulationen direkt nach der Punktion der Venen gegeben ist. Nachdem der SalzMaN-Test sich uns als geeignet erwies, eine herabgesetzte Thrombocytenklebrigkeit bei
Patienten mit einem Morbus v. Willebrand-Jürgens und bei niereninsuffizienten oder mit Acetylsalicylsäure behandelten Patienten zu erfassen, haben wir in der vorliegenden Arbeit mit diesem Test die Adhäsivität der Thrombocyten bei verschiedenen Patientengruppen, bei denen Gefäßerkrankungen gehäuft vorkommen oder bereits klinisch vorhanden waren, untersucht.

\section{Material und Methoden}

\section{Untersuchte Patienten}

Mit dem leicht modifizierten Salzman-Test wurde die Thrombocytenadhäsivität bei folgenden Patientengruppen bestimmt:

1. 19 Patienten mit Adipositas $\left(6 \% 13 \sigma^{7}\right)$, deren Körpergewicht mindestens $25 \%$ über dem Durchschnittsgewicht lag. Das Durchschnittsalter in dieser Gruppe betrug 35,7 Jahre. Zusätzliche Risikofaktoren für Gefäßerkrankungen bei klinisch erkennbarer Atherosklerose lagen bei diesen Patienten nicht vor.

2. a) 47 Patienten $\left(23 \%, 24 \delta^{3}\right)$ mit einem Hypertonus. Davon hatten $17\left(8\right.$ ㅇ, 9 $\left.0^{*}\right)$ weniger als 2 Jahre erhöhte Blutdruckwerte, der diastolische Wert war in keinem Fall höher als $110 \mathrm{~mm} \mathrm{Hg}$ (Durchschnittswerte 175/100, Durchschnittsalter 54,9 Jahre). Gefäßerkrankungen waren klinisch nicht zu erfassen.

b) 30 Patienten ( 15 o, $15 \delta^{\star}$ ) hatten einen länger als 2 Jahre bestehenden, meistens anbehandelten Hypertonus, die durchschnittlichen Blutdruckwerte betrugen 195/110 mm Hg. Das Durchschnittsalter war 55,6 Jahre. In dieser Gruppe waren häufig Zeichen einer bestehenden Atherosklerose klinisch oder röntgenologisch eindeutig zu erfassen.

Die Patienten in dieser Gruppe crhielten als antihypertensive Therapic Adelphan ${ }^{1}$ ) oder Presinol' ${ }^{2}$ ) in unterschiedlicher Dosierung.

1) Ciba; Reserpin + Dihydralazinsulfat.

2) Ciba; Dihydralazinsulfat. 
3. 41 Diabetiker $\left(26\right.$ \%, $15 \delta^{\circ}$ ), die bereits längere Zeit (mind. mehr als $1 \mathrm{Jahr}$ ) in unserer Diabetesambulanz betreut wurden.

In diescr Gruppe bestanden neben dem Diabetes mellitus häufig Gefäßkrankheiten, ein Hypertonus oder eine Adipositas, so daß eine Becinflussung durch Komplikationen des Diabetes mellitus oder aber durch Zweitkrankheiten gegeben ist.

Die untersuchten Patienten mit Altersdiabetes wurden zum Zeitpunkt der Untersuchung mit oralen Antidiabetika (Tolbutamid und/oder Buformin) behandelt.

4. Patienten mit Coronarerkrankung

a) 10 Männer mit Zustand nach Infarkt, weniger als $1 \mathrm{Jahr}$ zurückliegend. Das Durchschnittsalter betrug 56,8 Jahre. Alle diese Patienten wurden mit Antikoagulantien (Marcumar ${ }^{3}$ )) behandelt.

b) 24 Patienten $(1+, 23 \hat{\jmath})$, Durchschnittsalter 57,9 Jahre, hatten vor mehr als einem Jahr einen Herzinfarkt durchgemacht. 18 dieser 24 Patienten standen unter Antikoagulantien.

c) 18 Patienten $\left(5 \circ, 13 \delta^{7}\right)$ mit Angina pectoris, Durchschnittsalter 63,6 Jahre. In dieser Gruppe konnte weder klinisch, elektrocardiographisch noch nach den Laborbefunden ein Infarkt gesichert werden.

5. a) 12 Patienten $\left(5 q, 7 \delta^{\circ}\right.$ ), Durchschnittsalter 48,9 Jahre, mit einer akuten, ausgedehnten Venenthrombose, die bei der Messung der Thrombocytenadhäsivität nicht länger als 14 Tage bestand, wurden ebenfalls untersucht.

b) 9 Patienten $\left(2 q, 7 \delta^{\prime}\right)$, Durchschnittsalter 56,3 Jahre, mit mehr als 6 Monate zurückliegender Venenthrombose oder Nierenembolie.

6. 28 Patienten ( $11 \%, 17 \delta$ ), Durchschnittsalter 46,7 Jahre, mit Allgemeinkrankheiten ohne Hinweise auf Gefäßerkrankungen, wurden ebenfalls unțtersucht.

In dieser Gruppe waren im einzelnen folgende Erkrankungen zusammengefaßt: Von diesen Patienten hatten 5 eine chronische Emphysembronchitis, 5 Patienten einen frisch entdeckten, noch unbehandelten Diabetes mellitus, 4 Patienten eine Hyperthyreose, 3 Patienten ein Ulcus ventriculi, 2 Patienten einen Diabetes renalis, 2 Patienten ein LWS-Syndrom, 2 Patienten eine Gastroduodenitis und je ein Patient hatte einen Diabetes insipidus, eine Myocarditis, ein Maxillaneoplasma, ein Erythema nodosum und einmal ein Prostata-Adenom.

Thrombocytenadhäsivitätsmessung nach SALZMAN $(22,23)$ (modifiziert)

Eine Kontrollprobe Venenblut wurde durch eine „Einmalkanüle“ Pravaz Nr. 1 über den Adapter Nr. 3200 A in ein Vakuumröhrchen gezogen, das als Antikoagulans $0,2 \mathrm{ml}$ einer $80 \mathrm{~g} / 1$ EDTA-Lösung enthielt. An dieselbe Kanüle wurde anschließend über ein Verbindungsstück der Glasperlenfilter angeschlossen und das Blut nach Passage der Glasperlensäule ebenfalls in einem Vakuumröhrchen mit Antikoagulans gesammelt. Nach unseren Voruntersuchungen hatte eine Sammelzeit von genau $45 \mathrm{~s}$, die auch bei diesen Untersuchungen angewandt wurde, die am besten übereinstimmenden Werte mit einem nur geringen Streubereich bei 3-fach und 2-fach-Bestimmungen, erbracht. Die in dieser Zeit gesammelte Blutmenge betrug $6 \pm 0,5 \mathrm{ml}$, was einer Durchströmungsmenge von $8 \pm 0,6 \mathrm{ml} / \mathrm{min}$ entspricht.

Das Glasperlenfilter besteht aus einem Polyvinylschlauch $(0,113 \times$ 0,022 inch; Insulon Medical), der 1,3 g Glasperlen (Minnesota Mining Company Typ „Superbrite“, Durchmesser der einzelnen Perlen um 0,0185 inch) enthält. Der Schlauch wird an beiden Enden mit ausgestanzten Nylonsieben (Transfusionsgerät Fenwal HB 13-D) verschlossen, die dem Anschlußstück und dem Adapter $3200 \mathrm{~A}$ aufliegen. Da die ursprünglich benutzten B-D Vacutainer bei Messungen störende Unterdruckschwankungen aufwiesen $(17,19)$, wurde in den B-D Vacutainern der Unterdruck kurze Zeit vorher selbst hergestellt. Als Antikoagulans wurden $0,2 \mathrm{ml}$ einer $80 \mathrm{~g} / \mathrm{l}$ EDTA-Lösung $=16 \mathrm{mg}$ EDTA in physiologischer $\mathrm{NaCl}-\mathrm{L}$ ösung verwandt. Um für die nephelometrische Thrombocytenzählung (24), die in unserer Klinik seit mehr als 4 Jahren routinemäßig angewandt wird, ein möglichst einheitlich sedimentiertes plättchenteiches EDTA-Plasma zu gewinnen, wurde als Sedimentationsbeschleuniger Dextran 150 (Pharmacia

3) Hoffmann-La Roche; 3-(1'-Phenyl-propyl)-4-hydroxycumarin.
Uppsala) hinzugefügt mit einer Dextranendkonzentration im Blut von $2,5 \mathrm{~g} / \mathrm{l}$. Die Thrombocytenadhäsivität in $\%$ wurde exhalten, indem man von der normalen Thrombocytenzahl die nach der Glasperlenpassage erhaltene Thrombocytenzahl abzog und durch die Normalzahl dividierte.

Die von uns durchgeführte Modifikation des Salzman-Tests besteht demnach in der nephelometrischen Bestimmung der Thrombocytenzahl, in der genauen Einhaltung einer Passagezeit von $45 \mathrm{~s}$ und in dem unmittelbàr vor Versuchsbeginn selbst hergestellten gleichmäßigen Unterdruck der Vakquumröhrchen.

\section{Statistische Auswertung der Befunde}

Für alle Gruppen wurde der Mittelwert der Adhäsivitätsmessungen und die Standardabweichung berechnet. Die Prüfung der Meßergebnisse bei Normalpersonen und Patienten auf eine Normalverteilung erfolgte graphisch durch Auftragen der Summenhäufigkeit im Wahrscheinlichkeitsnetz. Um festzustellen, ob die Varianzen der Mittelwerte der untersuchten Gruppen nicht wesentlich von der Varianz der Normalpersonengruppe ab.weichen, wurde der F-Test angewandt. $\mathrm{Da}_{a}$ Normalverteilungen vorliegen und auch die Varianzen det Mittelwerte der einzelnen Gruppen nicht wesentlich voneinander abweichen, wurde zur Prüfung der Signifikanz der Mittelwertsdifferenzen zwischen Patientengruppen und Normalpersonen der $t$-Test verwendet.

\section{Ergebnisse}

\section{Normalwerte}

Als Normalpersonen wurden gesunde, nicht adipöse Blutspender und Kliniksangehörige gewählt. Untersucht wurden 26 Frauen und 32 Männer (Durchschnittsalter 30,2 Jahre). Der Mittelwert der Thrombocytenadhäsivität betrug $30,19 \pm 7,49 \%$. Die Mẹwerte waren nicht geschlechts- oder altersabhängig. In allerdings nur 7 Fällen wurden die Meßwerte in Zeiträumen von 10-83 Tagen wiederholt, dabei zeigten die Werte nur eine Schwankungsbreite von $4 \%$ und somit eine gute Reproduzierbarkeit (Abb. 1). Patienten, deren Thrombocytenzahl nicht im Normbereich der nephelometrischen Zählmethode (150000-350000) lag, wurden nicht ausgewertet.

Die Mittelwerte und Standardabweichungen der Thrombocytenadhäsivität der einzelnen Gruppen sind in Abbildung 2 graphisch dargestellt.

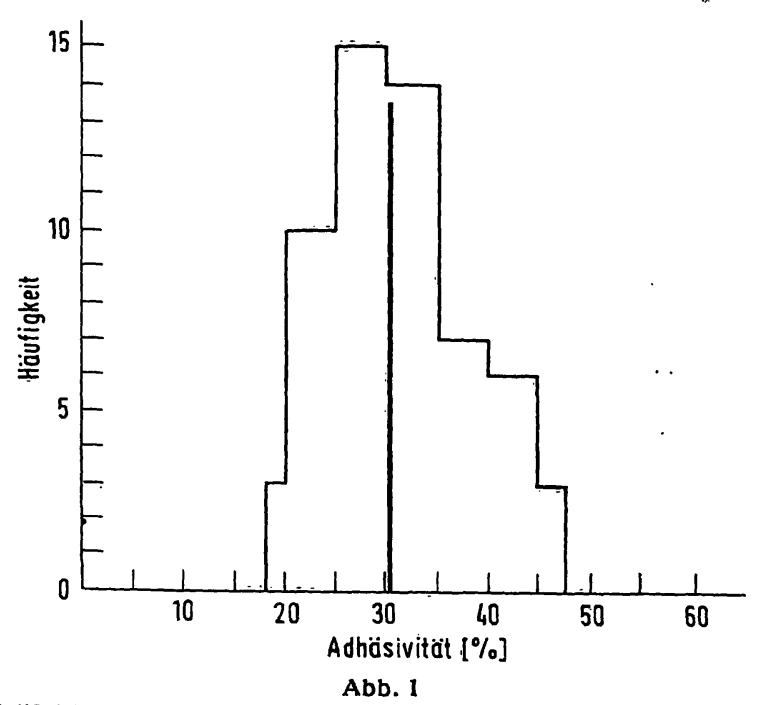

Bei 58 Normalpersonen mit dem SALzMAN-Test erhaltene Thrombocyten-Adhäsivität. Der Mittelwert $(n=58)$ ist durch die Linie 


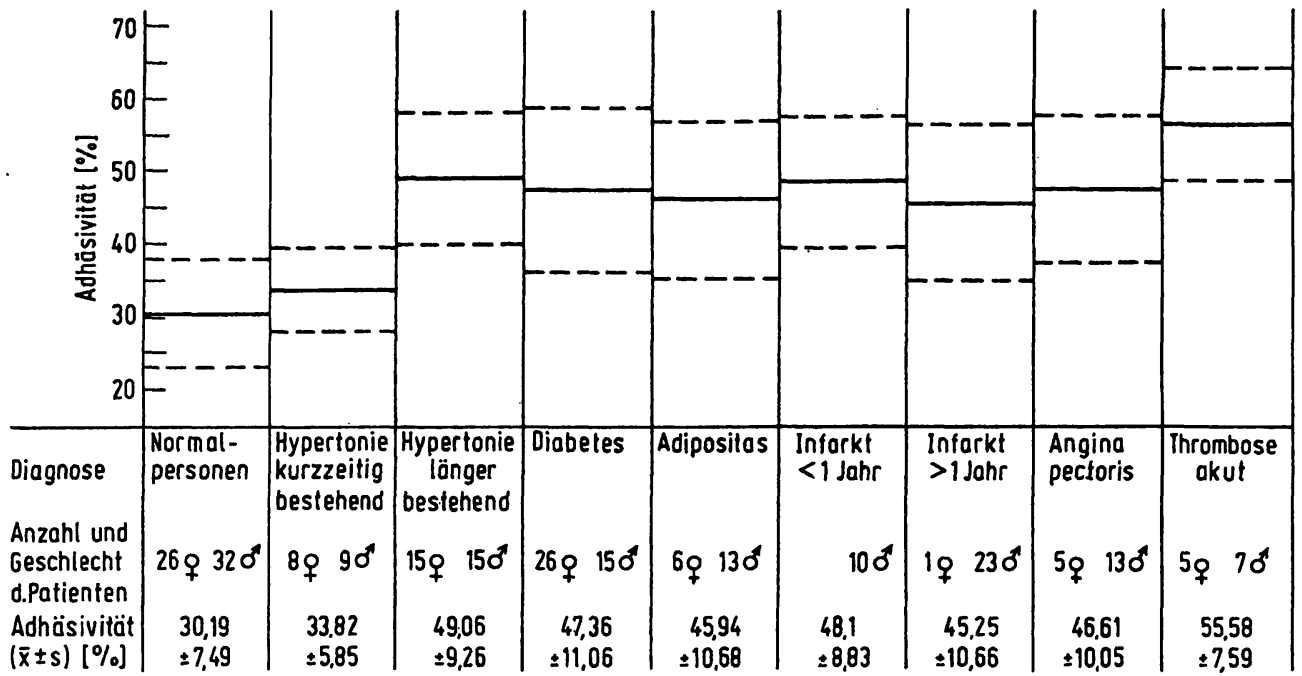

Abb. 2

Mittelwerte und Standardabweichungen aller mit dem SALZMAN-Test untersuchten Patienten-Gruppen in Relation zu den Normalpersonen
Bei den 19 Patienten mit einer Adipositas fand sich ein Mittelwert von 45,94 $\pm 10,68 \%$. Demnach ist die Thrombocytenadhäsivität signifikant gegenüber den Normalpersonen gesteigert.

Bei den 17 Patienten mit erst seit kurzer Zeit bestehendem Hypertonus ergab sich ein Mittelwert von 33,82 士 $5,82 \%$ und damit ein nicht signifikanter Unterschied zu der Normalgruppe.

Demgegenüber fand sich jedoch bei den 30 Patienten mit bereits seit längerer Zeit bestehendem Hypertonus in den Mittelwerten $(49,06 \pm 9,2 \%)$ ein signifikanter Anstieg der Thrombocytenadhäsivität. Diese Gruppe wurde in Abbildung 3 graphisch dargestellt.

Eine ähnliche Zunahme der Thrombocytenadhäsivität war auch bei den 41 Patienten mit länger bestehendem und behandeltem Diabetes mellitus festzustellen. Hier betrug der Mittelwert 47,36 $\pm 11,06 \%$. Er ist gegenüber den Normalpersonen signifikant gesteigert. Die graphische Darstellung der bei den einzelnen Patienten in dieser Gruppe gefundenen Einzelwerte zeigt jedoch, daß die Thrombocytenadhäsivität bei etwa $30 \%$ dieser Patienten noch im Normbereich liegt.

Bei 10 Patienten, deren Myocardinfarkt bei der Untersuchung weniger als $1 \mathrm{Jahr}$ zurück lag, fand sich ein Mittelwert von $48,1 \pm 8,83 \%$, bei 24 Patienten mit länger zurückliegendem Infarkt ein Mittelwert von

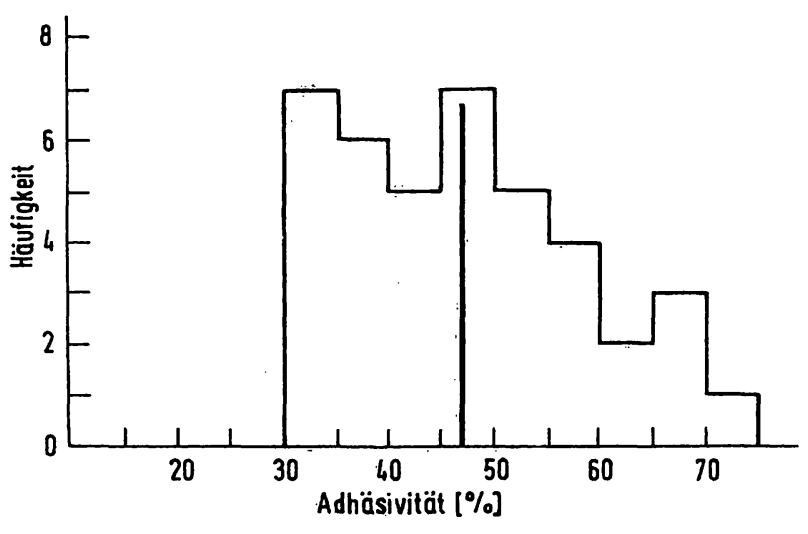

Abb. 3

Ergebnisse der Thrombocyten-Adhäsivitäts-Bestimmung von 30 Patienten, bei denen außer einem Hypertonus bereits Gefäßveränderungen macht gegenüber Abb. 1 die Zunahme der Adhäsivität deutlich
$45,25 \pm 10,66 \%$, unter 18 Patienten mit Angina pectoris ein Mittelwert von $46,61 \pm 10,05 \%$. Alle in diesen Gruppen bestimmten Mittelwerte sind gegenüber dem Normalwert signifikant erhöht.

Bei 12 Patienten mit einer nicht länger als 14 Tage zurückliegenden Thrombose wurde mit 55,58 $\pm 7,59 \%$ der höchste Mittelwert in allen Gruppen bestimmt, die Thrombocytenadhäsivität ist gegenüber Gesunden signifikant gesteigert.

Bei den 9 Patienten mit länger zurückliegenden Thrombosen oder Embolien wurde ein Mittelwert von $30,9 \pm 6,13 \%$ bestimmt, der nahezu gleich mit dem der Normalpersonen ist.

Bei den 28 Patienten, die keine Gefäßerkrankung erkennen ließen, fand sich ein Mittelwert von 29,82 士 $7,39 \%$. Auch dieser Wert stimmt mit dem der Gruppe der Normalpersonen überein.

Da bei allen Patienten der Cholesterinspiegel mitbestimmt wurde, wurde die gemessene Thrombocytenadhäsivität auch in Korrelation zur Höhe des Cholesteringehaltes im Serum gesetzt. Bei einem Cholesteringehalt bis $\mathrm{zu} 2,50 \mathrm{~g} / \mathrm{l}$ fanden sich sowohl erhöhte als auch erniedrigte Thrombocytenadhäsivitätswerte. Wenn auch bei einem erhöhten Cholesteringehalt die gemessene Thrombocytenadhäsivität meistens erhöht

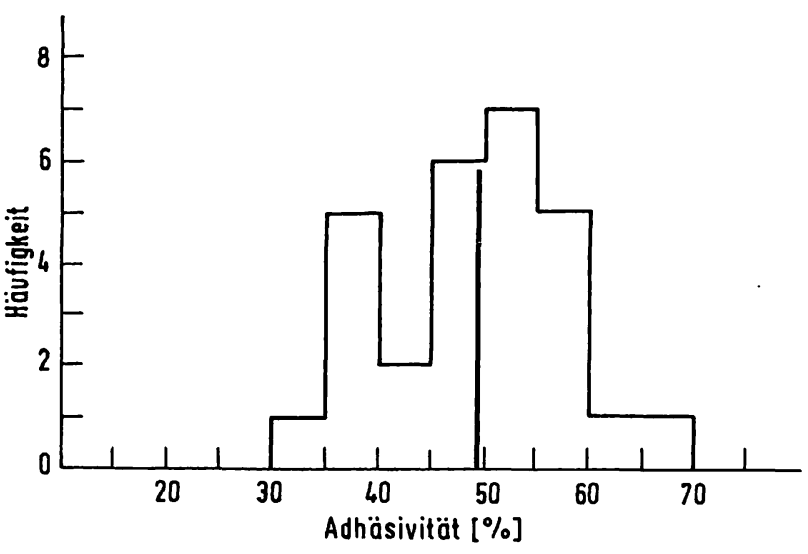

Abb. 4

Bei 41 Patienten mit länger bestehendem, behandlungsbedürftigem Diabetes mellitus bestimmte Thrombocyten-Adhäsivität. Auch hier ergab sich elne signifikante Zunahme des Mittelwertes (Linie) gegenüber den Normalpersonen. In dieser-Gruppe fand sich aber auch 
war, so zeigten jedoch auch Patienten, die nur eine Hypercholesterinämie und keine erkennbare Gefäßcrkrankung hatten, ausgesprochen niedrige Werte. Nach diesen Ergebnissen ergibt sich demnach kein sicherer Hinweis auf eine Übereinstimmung zwischen dem Cholesteringehalt im Serum und der mit dem SALZMAN-Test bestimmten Thrombocytenadhäsivität.

\section{Diskussion}

Wir behielten den von Salzman, der diese Methode zuerst beschrieb, gewählten Begriff des Adhäsivitätstestes (25) für unsere Untersuchungen bei, sind uns jedoch in Übereinstimmung mit anderen Autoren bewußt, daß mit diesem Test nicht nur die Adhäsion der Thrombocyten, sondern vielmehr auch die in der Pathogenese verschiedener Erkrankungen viel entscheidendere Aggregation der Thrombocyten aneinander erfaßt wird $(25,26)$. Daher ist die genaue Einhaltung der Durchströmungszeit der Glasperlénsäule von $45 \mathrm{~s}$, die mit den käuflichen Vacuumcontainern mit teilweise langer Lagerung nach unseren Erfahrungen nicht immer garantiert werden konnte, bei diesem Test, wie wir uns in zahlreichen Voruntersuchungen überzeugen konnten, besonders wichtig. Fin Unterschreiten oder Überschreiten dieser Zeit stellt nach diesen Voruntersuchungen die größte Fehlermöglichkeit beim SALZMran-Test dar. Alte Blutproben, bei denen die erhaltene Blutmenge von $6 \pm 0,5 \mathrm{ml}$ auch bei optimaler Passagezeit nicht erreicht oder überschritten wurde, wurden verworfen. Bei genauer Handhabung der Versuchsbedingungen $(27,28)$ sind reproduzierbare und verläßliche Ergebnisse zu erwarten. Bei häufig durchgeführten Doppel- und Dreifachbestimmungen fanden wir eine Schwankungsbreite von $4 \%$, andere (29) von $7 \% . \mathrm{Daß}$ andere (30) keine reproduzierbaren Werte fanden, dürfte vor allem in der Schwierigkeit begründet liegen, die Versuchsbedingungen konstant zu halten. Der von uns gefundene Normalbereich entspricht ziemlich genau auch dem von MEYER und LARRIEU (31). Zusammenfassend kann nach unseren Erfahrungen mit den hier ausgewerteten 256 Untersuchungen gesagt werden, $\mathrm{da} \beta$ es sich beim SALZMAN-Test um einen etwas schwierig durchzuführenden Test handelt, der aber bei strikter Beachtung der Untersuchungsbedingungen und bei konstanter Passagezeit verläßliche und gut reproduzierbare Werte liefert.

Für atherosklerotische Gefäßkrankheiten sind Risikofaktoren (32) bekannt. Bei einigen Patientengruppen, bei denen diese Risikofaktoren gegeben sind, wurde das Verhalten der Thrombocyten im SaLzMan-Test gemessen, da ein Fortschreiten der Gefäßkrankheit durch eine gesteigerte Thrombocytenfunktion möglicherweise mit unterhalten werden könnte und dann durch die Gabe von Aggregationshemmern (z. B. Acetylsalicylsäure-Präparate) $(32,33)$ günstig zu beeinflussen wären. Denkbar ist jedoch auch, daß diese gesteigerte Thrombocytenklebrigkeit eine notwendige Reaktion oder Anpassung der Thrombocyten an die atherosklerotisch veränderte Gefäßwand darstellt. Eine
Deutung dieses Problems läßt unser Versuchsansatz einer einmaligen Untersuchung der Thrombocytenfunktion nicht zu. Hierzu wären fortlaufende Untersuchungen dieser Patientengruppen notwendig. Nach unseren Ergebnissen besteht bei den von uns untersuchten Patienten mit einer Adipositas bereits bei einem recht niedrigen Durchschnittsalter und ohne klinisch erfaßbare Gefäßerkrankungen eine signifikant gesteigerte Thrombocytenadhäsivität. D'ieser Befund wurde auch von anderen (35) beschrieben. Ob die Ursache für diese Funktionssteigerung der Thrombocyten in der von Mog^ (36) gefundenen Dyslipämie mit einer Vermehrung der Gesamtlipide, einer Zunahme der freien Fettsäuren, einer Erhöhung des Cholesterinspiegels und $\operatorname{der} \beta$-Serumlipoproteinfraktion liegt, kann noch nicht entschieden werden. Bei den Patienten mit einem Hypertonus geht die Thrombocytenfunktionssteigerung in etwa parallel mit der Dauer des Hypertonus und den vorhandenen Gefäßerkrankungen. Obwohl das durchschnittliche Lebensalter in beiden Gruppen nahezu gleich ist, konnte bei den Patienten, deren Hypertonus erst kurz vorher festgestellt worden war, kein wesentlicher Unterschied zu den Normalpersonen festgestellt werden, jedoch ein signifikanter Anstieg in der ver-. gleichbaren Gruppe. Daraus folgt u. W., daß die Thrombocytenadhäsivität nicht korreliert ist zum Grad des Hypertonus, sondern eher zu den als Begleiterkrankung gehäuft auftretenden atherosklerotischen Gefäßveränderungen.

Ähnlich verhielt sich die Thrombocytenadhäsion auch bei den Patienten mit einem Diabetes mellitus. Bei allerdings nur 5 Patienten, deren Diabetes mellitus erst gerade erfaßt worden war und die bis zu unserer Untersuchung nur diätetisch behandelt worden waren, fand sich keine Abweichung zu der Gruppe der Normalpersonen. Bei längere Zeit bestehendem Diabetes mellitus und klinisch oft schon erfaßbaren Gefäßveränderungen kam es nach den Ergebnissen des SALZMaNTests zu einem signifikanten Anstieg der Thrombocytenadhäsivität. Trotzdem muß darauf hingewiesen werden, daß bei etwa $33 \%$ der Patienten in dieser Gruppe die gemessenen Werte noch im Normbereich liegen.

Eine Abhängigkeit zu der Höhe des gleichzeitig mitbestimmten postprandialen Blutzuckers $(37,38)$ ergab sich auch nach unseren Ergebnissen nicht. Mit verschiedenen Bestimmungsmethoden wurde auch von anderen Autoren (37-40) eine Steigerung der Thrombocytenadhäsivität bei Patienten mit einem Diabetes mellitus gefunden.

Nicht untereinander, wohl aber signifikant gesteigert gegenüber den Normalpersonèn waren die Ergebnisse der Thrombocytenadhäsivitätsmessungen bei den 3 Gruppen det Patienten mit Coronarerkrankungen, Befunde, die im wesentlichen auch von anderen Untersuchern erhoben wurden (41-45). Leider gelang es nicht, diese Messungen auch bei Patienten mit akutem Infarktereignis durchzuführen; alle Patienten dieser Gruppen mit Herzinfarkt wurden, teilweise schon sehr 
lange, mit Antikoagulantien behandelt. Nach anderen Autoren (44, 45), denen auch unsere Ergebnisse entsprechen, findet man nach Applikation von Cumarinderivaten keine Verringerung der Adhäsivität. $\mathrm{Da}$ bei allen Infarkt-Patienten die gemessenen Adhäsivitäten sehr hoch sind, kann dennoch allein aus einem einzelnen hohen Meßwert keine Vorhersage auf einen abermals bevorstehenden thrombotischen Verschluß im Bereich der Coronararterien abgeleitet werden. Die Adhäsivitätsmessungen der Thrombocyten sind u. E. nur geeignet, eine generelle Feststellung der tatsächlichen Verhältnisse bei bestimmten Erkrankungen zu geben, nicht jedoch im Einzelfall auch eine Prognose auf eine bevorstehende akute Verschlechterung.

Die höchsten von uns gemessenen Adhäsivitätswerte fanden sich bei Patienten mit relativ frischen Thrombosen (46-48). Es ergab sich jedoch bei diesen Erkrankungen eine gewisse Abhängigkeit der gesteigerten Thrombocytenfunktion von der Dauer des zurückliegenden thrombembolischen Ereignisses. 6Monate nach dieser Erkrankung glichen die gemessenen Werte bei den Patienten in dieser Gruppe bereits denen der Normalpersonen.

$\mathrm{Da}$ eine prinzipielle Gleichartigkeit der Pathogenese arterieller und venöser Thromben (49) besteht, wurden zurückliegende embolische Ereignisse dieser Erkrankungsgruppe zugeordnet.

Die abschließend erwähnten 28 Patienten mit verschiedenen Erkrankungen zeigten eine normale Thrombocytenadhäsivität.

Es fand sich auch keine Korrelation zwischen dem Cholesteringehalt und den gefundenen Werten der Thrombocytenadhäsivität (50), die auch nach Tierversuchen (51) nicht besteht. Vielmehr dürfte es sich um eine bei Gefäßerkrankungen häufig gleichzeitig bestehende Erhöhung sowohl der Thrombocytenadhäsivität als auch des Cholesteringehaltes im Serum handeln. Nach unseren Ergebnissen findet sich bei Patienten mit einer Adipositas, länger bestehendem Hypertonus und Diabetes mellitus, mit Zustand nach Infarkt, mit Angina pectoris und unmittelbar nach thrombembolischen Ereignissen eine signifikante Steigerung der Thrombocytenadhäsivität im SALZMAN-Test. Sie dürfte wahrscheinlich durch die Gefäßveränderungen hervorgerufen und unterhalten werden. $\mathrm{Ob}$ es überzeugend gelingt, diese pathologisch gesteigerte Thrombocytenfunktion durch Medikamente zu normalisieren oder so zu verringern, daß dadurch ein günstiger Effekt auf das Fortschreiten der Gefäßerkrankungen erreicht werden kann, bleibt abzuwarten.

\section{Literatur}

1. Bizzozero, V. (1882), Virchows Arch. Pathol. Anat. 90, 261-332. - 2. Eberth, C. J. \& Schimmelbusch, C. (1888), Enke-Verlag Stuttgart. - 3. RoKrtanskY, C. von: Braunmüller und Seidel, Wien 1841-46. - 4. Duguid, J. B. (1948), J. Pathol. Bact. 60, 57-61. - 5. Evans, G. \& IRVINE, W. T. (1966), Lancet II, 353-355. - 6. Mustard, J. F., Packham, M. A., Roswell, H. C. \& Jørgensen, L. (1967), Thromb. Diath. Hämorrh. Suppl. XXVI, 261-274. - 7. BREDdIN, K. (1968), Deut. Med. Wochenschr. 93, 1555-1565. - 8. HeLLEM, A. H. (1960), Scand. J. Clin. Lab. Invest. 12, Suppl. 51. - 9. Hume, M. (1966), Surgery 59, 110-114. - 10. Marx, R. (1961), Folia Hämatol. (Leipzig) N. F. 6, 337-340. - 11. Moolten, S. E. \& Vroman, L. (1949), Amer. J. Clin. Pathol. 19, 701-709. - 12. Murphy, E. A. \& Mustard, J. F. (1960), Circ. Res. 8, 1187-1199. - 13. O'Brien, J. R. (1961), J. Clin. Pathol. 14, 140-149. - 14. Poliwoda, H. (1969), 13. Tagung des DAB Stuttgart. - 15. WRIGirT, H. P. (1941), J. Pathol. Bact. 53, 255-262. - 16. Adelson, E., KaurMAN, R. M., Lear, A. A., Krrby, J. C. \& Rheingold, J. J. (1963), J. Lab. Clin. Med. 62, 385-393. - 17. BorN, G. V. R. \& Cross, M. J. (1963), J. Physiol. 168, 178-195. - 18. Gross, R., Niemeyer, G. \& Reuter, H. (1969), Deut. Med. Wochenschr. 94, 1735-1743. - 19. Murphy, E. A. \& Mustard, J. F. (1962), Circulation $25,114-125$. - 20. Niemeyer, G. \& ReUter, $H$. (1968), Internist 9, 48-55. - 21. LECHNER, K. (1972), 16. DABTagung Bonn. - 22. SALzMAX, E. W. (1967), Thromb. Diath. Hämorrh. Suppil. XXVV; 303-307. - 23. SALZMAN, E. W. (1263), J. Lab. Clin. Med. 62, 724-735. - 24. Schulz, V., Kochsiek, K., Köstering, H. Walther, Chr. (1971), diese Z. 9, 324-328. 25. Salzman, E. W. Persönliche Mitteilung. - 26. Mannucr, P. M. \& Sharp, A. A. (1967), Brit. J. Hämatol. 13, 604-61727. Hartmann, R. C. (1966), Thromb. Diath. Hämorrh. Suppl. XXVI, 281-302. - 28. MeYer, D. \& Larrieu, M. J. (1967), Rev. Fr. Etud. Clin. Biol. 12, 736-739. - 29. Srrauss, H. S. \&
Bloom, G. E. (1965), N. Engl. J. Med. 273, 171-181. - 30. Baumgartiner, H. R., Cronquist, M., Wobmann, P., Streuli, F. \& Duckert, F. (1967), Schweiz. Med. Wochenschr. 97, 1674-1679. - 31. Meyer, D. \& Larrieu, M. J. (1967), Rev. Fr. Etud. Clin. Biol. 12, 736-739. - 32. Epstern, F. H. (1965), J. Chronic Dis. 18, 735-774. - 33. Al-Mondhiry, H., Marcus, A. J. \& Spaet, T. H. (1970), Proc. Soc. Exper. Biol. (N. Y.) 133, 632-636. - 34. Werss, H. J. (1970), Blood 35, 333-340. 35. Gavrilescu, S., Bartl, F., Csernik, I., Anghelescu L. \& MogA, A. (1967), Deut. Z. Verdau.- u. Stoffwechselkr. 27, 191-195. - 36. Moga, A., Cucuianu, M., Crisnic, I. \& Olinic, N. (1966), Rev. Roum. Med. Int. 3, 407-412. - 35. ShAw, S., Pegrum, G. D. Wolff, S. \& Ashton, W. L. (1967), J. Clin. Pathol. 20, 845-847. - 38. MAYNE, E. E. (1968), Vierte Tagung, Luvain, Belgien. - 39. Breddin, K. (1968), Deut. Med. Wochenschr. 93, 1555-1565. - 40. BRIDGes, J. M., DABY, M., Millar, J. H. D. \& Weaver, J. A. (1965), Lancet I, 75-77. 41. BreddiN, K. (1964), Thromb. Diath. Hämorrh. 12, 269-281. - 42. BrednIN, K. (1968), Deut. Med. Wochenschr. 93, 1555-1565. - 43. Moolten, S. E. \& VRoman, L. (1949), Amer. J. Clin. Pathol. 19, 701-709. - 44. Baumgartner, H. R., Cronguist, M., Wobmann, P., Streuli, F. \& Duckert, F. (1967), Schweiz. Med. Wochenschr. 97, 1674-1679. 45. PFleiderer, TH. \& Rücker, G. (1964), Klin. Wochenschr: 42, 1223-1226. - 46. BredDIN, K. (1964), Thromb. Diath. Hêmorirh. 12, 269-281. - 47. Hirsh, J., McBridGe, J. A. \& WRIGHT, H. P. (1966), Thromb. Diath. Hämorrh. 16, 100-104 48. Hume, M. (1966), Surgery 59, 110-114. - 49. Loo, J. vaN DE (1968), Med. Klin. 63, 361--366. - 50. Moga, A., Cucuianu, M., Crisnic, I. \& Olinic, N. (1966), Rev. Roum. Méd. Int. 3, 407-412. - 51. Jipp, P. \& JACOBSON, F. (1967), Z. Kreisl. Forsch. 56, 1550-1556.
Priv.-Doz. Dr. H. Köstering Med. Klinik Blutgerinnungslab. 34 Göttingen 